\title{
Escala Diagramática para a Quantificação do Complexo de Doenças Foliares de Final de Ciclo em Soja*
}

\author{
Mônica C. Martins ${ }^{1 * *}$, Rodrigo A. Guerzoni ${ }^{1}$, Gil M. de S. Câmara' ${ }^{1}$ Patrícia Mattiazzi ${ }^{1}$, Sílvia A. \\ Lourenço $^{3}$ \& Lilian Amorim ${ }^{3}$

\begin{abstract}
${ }^{1}$ Departamento de Produção Vegetal, Escola Superior de Agricultura "Luiz de Queiroz”, Universidade de São Paulo, Cx. Postal 9, CEP 13418-900, Piracicaba, SP, e-mail: mcmartin@esalq.usp.br e fundacaoba.soja @aiba.com.br; ${ }^{2}$ Departamento de Entomologia, Fitopatologia e Zoologia Agrícola, Escola Superior de Agricultura "Luiz de Queiroz", Universidade de São Paulo, Cx. Postal 9, CEP 13418-900, Piracicaba, SP
\end{abstract}

(Aceito para publicação em 06/01/2004)

Autor para correspondência: Mônica C. Martins

MARTINS, M.C., GUERZONI, R.A., CÂMARA, G.M.S., MATTIAZZI, P., LOURENÇO, S.A. \& AMORIM, L. Escala diagramática para a quantificação do complexo de doenças foliares de final de ciclo em soja. Fitopatologia Brasileira 29:179-184. 2004.

\section{RESUMO}

A ocorrência das doenças foliares de final de ciclo em soja (Glycine max) causadas pelos fungos Septoria glycines e Cercospora kikuchii é facilmente observada no campo. Entretanto, são necessárias informações precisas sobre a quantificação de danos e perdas na produtividade. A falta de um método padrão de quantificação visual para essas doenças pode levar a estimativas imprecisas da severidade das mesmas, induzindo a conclusões erradas. Com o objetivo de elaborar uma escala diagramática para quantificar a severidade dessas doenças, foram coletadas em campo, folhas apresentando diferentes níveis de severidade. A área de cada folha e sua correspondente severidade foram determinadas e, obedecendo-se a "Lei do estímulo de Weber-Fechner", foi elaborada uma escala com os níveis de severidade de doença: 2,4; 15,$2 ; 25,9 ; 40,5$ e $66,6 \%$. A validação foi realizada por nove avaliadores, sem experiência na avaliação das doenças de final de ciclo, os quais estimaram a severidade de 30 folhas de soja com sintomas destas doenças. A precisão das avaliações variou de acordo com o avaliador $\left(0,84<\mathrm{R}^{2}<0,65\right)$, bem como a acurácia $(0,00<a<3,40 ; 0,90<b<1,29)$, não ocorrendo erro sistemático na superestimativa ou subestimativa da doença entre os avaliadores, demonstrando que a escala desenvolvida é adequada para a avaliação das doenças de final de ciclo em soja.

Palavras-chave adicionais: Glycine max, mancha parda, crestamento foliar de cercospora, patometria, severidade.

\section{ABSTRACT}

\section{Diagrammatic scale for assessment of late season leaf diseases} in soybean

The occurrence of late season leaf diseases in soybean (Glycine max) caused by the fungi Septoria glycines and Cercospora kikuchii are easily identified in field. However, there is a need for more precise information to quantify damage and yield losses. The lack of a standardized visual method may lead to inaccurate estimates of severity and innaccurate conclusions. With the purpose of elaborating a diagrammatic scale to assess these diseases, leaves with different levels of severity were collected in the field. The area of each leaf and its correspondent severity were

\section{INTRODUÇÃO}

A mancha parda ou septoriose causada pelo fungo Septoria glycines Hemmi e o crestamento foliar de cercospora e a mancha púrpura da semente causadas pelo fungo Cercospora kikuchii (Matsu. \& Tomoyasu) Gardner são doenças que estão presentes em todas as regiões produtoras de soja (Glycine max L.) do Brasil. Por ocorrerem na mesma época e devido às

\footnotetext{
* Parte da Tese de Doutorado do primeiro autor e da Dissertação de Mestrado do segundo autor. Escola Superior de Agricultura "Luiz de Queiroz" (2003)

** Bolsista FAPESP
}

determined, and following the "Stimulus Law by Weber-Fechner", a scale was elaborated with severity levels: 2.4, 15.2, 25.9, 40.5 and $66.6 \%$. Validation was carried out by nine appraisers, without previous practice in assessing late season leaf diseases, who estimated the severity on 30 leaflets of soybean (Glycine max) with disease symptoms. The variance found among appraisers $\left(0.84<\mathrm{R}^{2}<0.65\right)$, and accuracy $(0.00<\mathrm{a}<3.40 ; 0.90<\mathrm{b}<1.29)$, did not constitute systematic mistakes in over or underestimating the severity of disease. The developed diagrammatic scale is, therefore, appropriate to quantify late soybean season disease severity dificuldades que apresentam nas avaliações individuais, são consideradas como um "complexo de doenças de final de ciclo" (DFC), podendo reduzir a produtividade da soja em mais de 20\% (Lim, 1989; Yorinori, 1998; Embrapa, 2000).

Os sintomas causados por $S$. glycines aparecem nas nervuras das folhas ou muito próximos a elas, como manchas de coloração castanho-avermelhada que geralmente são observadas nas folhas inferiores. À medida que as plantas se aproximam da maturação, a doença progride rapidamente para as partes superiores (Ferreira et al., 1979; FAO, 1995). As lesões causadas por $C$. kikuchii podem começar como minúsculas manchas de coloração marrom-arroxeada, que se expandem 


\section{M.C. Martins et al.}

irregularmente e, assim como as da mancha parda, podem coalescer, necrosando extensas áreas do limbo foliar. O sintoma mais evidente de $C$. kikuchii é observado nas folhas superiores e jovens, que tornam-se coriáceas e, se expostas ao sol, exibem uma tonalidade púrpura (Ito \& Tanaka, 1993). Nos estádios finais do ciclo da cultura, essas doenças ocorrem concomitantemente, causando a queda prematura das folhas provocada pelo amarelecimento e necrose, podendo acelerar a maturação (Ferreira et al., 1979; Ito \& Tanaka, 1993).

A importância das DFC nesta cultura justifica a preocupação de pesquisadores e produtores em estudar medidas de controle e determinar a eficiência de fungicidas para estas doenças. Para isso, sua quantificação é necessária, pois, somente quando uma doença é mensurada corretamente é que se pode demonstrar o quanto de perda ela ocasiona (Horsfall \& Cowling, 1978). No caso de doenças foliares como manchas, a severidade, porcentagem da área de tecido coberto por sintomas, retrata melhor a quantidade de doença que a incidência (Amorim, 1995).

Para a quantificação da severidade de doenças com precisão várias estratégias têm sido propostas e entre estas, destacam-se as escalas diagramáticas, que são representações ilustradas de uma série de plantas, folhas ou partes de plantas com sintomas em diferentes níveis de severidade (Bergamin Filho \& Amorim, 1996). Atualmente, as escalas diagramáticas têm-se constituído na principal ferramenta de avaliação de severidade para muitas doenças. As escalas devem ser de fácil uso, aplicáveis a uma grande faixa de diferentes condições, ter resultados reproduzíveis, possuir intervalo suficiente para representar todos os estágios de desenvolvimento da doença, e permitir uma avaliação imediata (Berger, 1980). Assim, na sua elaboração, devem-se considerar aspectos como, os limites superiores e inferiores, os quais devem corresponder, respectivamente, à quantidade máxima e mínima da doença encontrada no campo; a representação dos sintomas, devendo estar tão próxima quanto possível àqueles observados na planta; e os níveis intermediários da severidade da doença, considerando as limitações de acuidade da visão humana, como definidas pela "Lei do estímulo de Weber-Fechner" (Horsfall \& Barrat, 1945; Nutter Jr. \& Schultz, 1995). Além disto, as escalas diagramáticas devem ser testadas (validadas) antes de serem propostas como um método padrão de quantificação de doenças e, no caso de produzirem resultados insatisfatórios, estas devem ser corrigidas.

Devido à ausência de métodos padronizados para a quantificação das DFC da soja e da importância deste complexo de doenças para esta cultura, este trabalho teve como objetivo, elaborar e validar uma escala diagramática para avaliação da severidade destas doenças.

\section{MATERIAL E MÉTODOS}

\section{Elaboração da escala diagramática}

A escala diagramática para avaliação das DFC da soja foi elaborada a partir de 100 folhas coletadas dos cultivares de soja MG/BR 46 (Conquista), BRS 133 e IAC 18, que apresentavam diferentes níveis de severidade dessas doenças. Após essa coleta, a área lesionada (tecido necrosado e o halo amarelado) de cada folíolo foi desenhada em plástico transparente, o qual foi submetido ao medidor de área foliar LICOR (LI-3000) para determinação das áreas lesionada e total de cada um destes. Dessa forma, foi possível determinar o folíolo de soja com menor número de lesões e o com maior número, estabelecendo assim, o limite inferior e o superior da escala diagramática, respectivamente. Além dos níveis mínimo e máximo, foram determinados matematicamente mais três níveis intermediários de acordo com a acuidade da visão humana, definida pela "lei do estímulo de Weber e Fechner" (Horsfall \& Barratt, 1945).

Uma vez estabelecidas as porcentagens das doenças a serem utilizadas na escala, um folíolo padrão de área conhecida foi representado cinco vezes, começando do nível mínimo, seguindo um incremento logístico de severidade, de maneira a permitir a reprodução dos sintomas observados nas folhas em campo, estabelecendo assim, a escala diagramática.

Na época em que a escala diagramática foi elaborada, as folhas apresentavam lesões agrupadas, porém, observou-se em campo, posteriormente, folhas com lesões esparsas. Dessa forma, a escala diagramática foi construída para representar os dois tipos de sintomas. Para cada nível de severidade há duas representações de folíolos: com sintomas agregados ou aleatoriamente distribuídos.

\section{Validação da escala diagramática}

A validação da escala proposta foi realizada a partir de 30 folíolos de soja com diferentes níveis de severidade das DFC que foram distribuídos para nove avaliadores sem experiência na avaliação destas doenças. Utilizando a escala diagramática desenvolvida, estimou-se a severidade das doenças nos folíolos considerando como área doente o tecido necrosado e os halos amarelados. A severidade real dos 30 folíolos foi determinada a partir de desenhos da área lesionada em plástico transparente, considerando-se o tecido necrosado e o halo amarelado de cada folíolo. Cada plástico com as lesões bem como seus correspondentes folíolos foram submetidos três vezes ao medidor de área foliar LICOR (LI-3000) para determinação das áreas lesionada e total de cada folíolo, respectivamente, obtendo-se desta forma, a severidade real (porcentagem de área foliar lesionada) dessas doenças.

A precisão e a acurácia das estimativas visuais de cada avaliador foram determinadas por meio de análise de regressão linear, considerando a severidade real como variável independente e a severidade estimada como variável dependente. A precisão das estimativas foi avaliada pelo coeficiente de determinação da regressão $\left(\mathrm{R}^{2}\right)$ e pela variância dos erros absolutos (severidade estimada menos severidade real). A acurácia de cada avaliador foi determinada por meio do teste $t$ aplicado ao coeficiente angular da reta $(b)$ e ao coeficiente linear da reta $(a)$, ambos obtidos pela regressão linear (severidade real $\mathrm{x}$ severidade estimada).

A reprodutibilidade da escala foi avaliada pelos coeficientes de determinação das regressões lineares entre as 
Escala diagramática para a quantificação do complexo de doenças...

severidades estimadas dos diferentes avaliadores, combinados em pares, como proposto por Nutter Jr. \& Schultz (1995) e Nutter Jr. et al. (1993).

\section{RESULTADOS E DISCUSSÃO}

A escala diagramática elaborada neste trabalho, com cinco níveis de severidade, teve como limite inferior e superior os valores de 2,4\% e 66,6\% de área lesionada, respectivamente (Figura 1). Valores de severidade acima de 66,6\% não foram incluídos na escala diagramática, pois estes não foram observados em campo uma vez que, levam as folhas à senescência.

O incremento logarítmico nos níveis de severidade é uma das características das escalas responsáveis pela fácil interpolação nas estimativas de severidade (Godoy et al., 1997). Estes incrementos são freqüentemente denominados como um sinônimo da "Escala de Horsfall \& Barratt". Quando esses autores redescobriram a "Lei de Weber-Fechner" (Horsfall \& Barratt, 1945), propuseram uma escala diagramática com níveis seguidos de severidade: $1(0), 2(0 \%-3 \%), 3(3 \%-6 \%), 4(6 \%-$ $12 \%), 5$ (12\%-25\%), 6 (25\%-50\%), 7 (50\%-75\%), 8 (75\%$88 \%), 9(88 \%-94 \%), 10$ (94\%-97\%), $11(97 \%-100 \%)$ e 12 (100\%). Fitopatologistas (Forbes \& Korva, 1994; Nutter Jr. \& Schultz, 1995) têm hesitado em usar tal escala, principalmente devido ao grande intervalo apresentado nas severidades médias, com apenas dois níveis entre $25 \%$ e $75 \%$ de severidade de doença (Campbell \& Madden, 1990). Entretanto, esses níveis médios podem ser subdivididos em intervalos menores, como proposto por Amorim et al. (1993), e como utilizado neste trabalho. É importante ressaltar que a escala diagramática deve seguir a "Lei de Weber-Fechner" (incrementos logarítmicos), sem necessariamente utilizar intervalos escolhidos por Horsfall $\&$ Barratt (1945).

Para que pesquisadores e produtores de soja possam
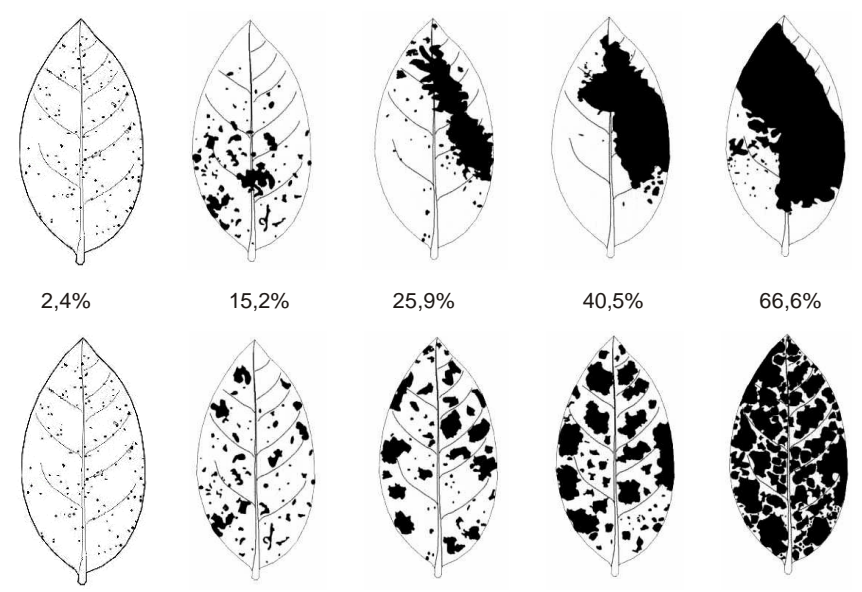

FIG. 1 - Escala diagramática das doenças de final de ciclo da soja (Glycine max) causadas por Septoria glycines e Cercospora kikuchii. Painel superior: Sintomas agregados. Painel inferior: Sintomas aleatoriamente distribuídos utilizar esta escala, fez-se necessário sua validação. Para a maioria dos avaliadores, os valores estimados de severidade ficaram próximos dos valores da severidade real (Figura 2). Esta proximidade entre a estimativa e a realidade determina a acurácia das avaliações, termo este, definido como a exatidão de uma medida isenta de erros sistemáticos, a qual pode ser medida pelo coeficiente angular $(b)$ e o intercepto da regressão linear $(a)$ entre severidade real e estimada (Bergamin Filho \& Amorim, 1996). Desta forma, avaliadores considerados acurados têm a média das estimativas de severidade próximas da média real. Para isto ocorrer, de acordo com Nutter Jr. et al. (1993), a inclinação da regressão linear $(b)$ deve ser igual a um (1), sem desvios sistemáticos e o intercepto $(a)$ deve ser igual a zero (0).

A maioria dos avaliadores que utilizou esta escala, pode ser considerada acurada pois, em suas avaliações, os valores de $a$ não foram significativamente diferentes de zero (0) e os valores de $b$ não foram significativamente diferentes de um (1). As estimativas de severidade ficaram muito próximas da severidade real. Exceção a estes, foram os avaliadores 5, 6 e 9, para os quais constatou-se valores de $b$ significativamente diferentes de um (1) (Tabela 1). O avaliador 5 subestimou a severidade das doenças foliares de final de ciclo, uma vez que o coeficiente angular da regressão linear entre as severidades real e estimada foi significativamente menor que 1 , enquanto que os avaliadores 6 e 9, superestimaram a severidade dessas doenças, uma vez que esses mesmos coeficientes foram significativamente maiores do que 1 (Figura 2 e Tabela 1).

Além da acurácia, a precisão, definida como a exatidão de uma operação onde há rigor ou refinamento na medida (Bergamin Filho \& Amorim, 1996) é um fator a ser considerado na validação de uma escala diagramática. A precisão pode ser avaliada por meio do coeficiente de determinação da regressão, que deve ser próximo de $100 \%$, e pela variação dos erros absolutos (diferenças entre severidades estimada e real) (Nutter Jr. \& Schultz, 1995). Utilizando a escala desenvolvida para as DFC, os avaliadores, com exceção do 4, apresentaram boa precisão em suas avaliações, com coeficientes de determinação, entre 0,71 e 0,84 (Tabela 1). Além do $R^{2}$, a boa precisão dos avaliadores foi confirmada quando se efetuou a diferença entre

TABELA 1 - Coeficientes lineares $(a)$, coeficientes angulares $(b)$ e coeficientes de correlação $\left(R^{2}\right)$ obtidos nas regressões entre severidade real (variável independente) e severidade estimada (variável dependente) de 30 folíolos de soja (Glyxine max) com doenças de final de ciclo, avaliados por nove avaliadores utilizando a escala diagramática proposta

\begin{tabular}{cccccccccc}
\hline \multirow{2}{*}{ Coeficiente } & \multicolumn{10}{c}{ Avaliadores } \\
\cline { 2 - 10 } & $\mathbf{1}$ & $\mathbf{2}$ & $\mathbf{3}$ & $\mathbf{4}$ & $\mathbf{5}$ & $\mathbf{6}$ & $\mathbf{7}$ & $\mathbf{8}$ & $\mathbf{9}$ \\
\hline$a$ & 1,97 & 1,65 & $-1,34$ & $-2,69$ & 0,50 & $-1,76$ & 0,00 & $-0,18$ & $-3,40$ \\
$b$ & 0,91 & 1,00 & 1,19 & 1,25 & $0,82^{*}$ & $1,27 *$ & 1,15 & 0,90 & $1,29 *$ \\
$R^{2}$ & 0,75 & 0,84 & 0,84 & 0,65 & 0,84 & 0,80 & 0,71 & 0,82 & 0,79 \\
\hline
\end{tabular}

* situações nas quais as hipóteses $a=0$ ou $b=1$ foram rejeitadas pelo teste $\mathrm{t}$ a $5 \%$ de significância. 

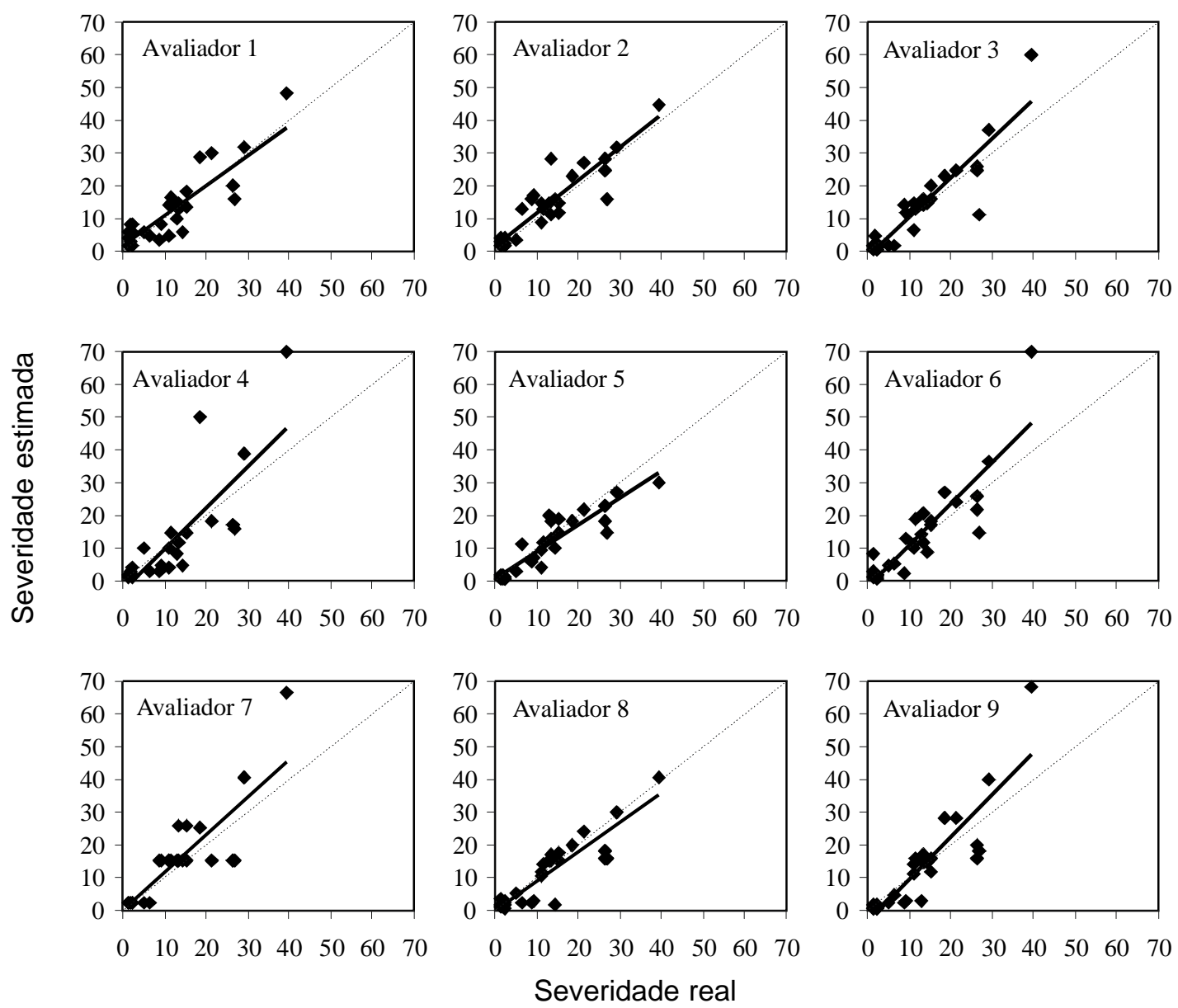

FIG. 2 - Severidade estimada com o auxílio da escala diagramática elaborada (pontos cheios) e linhas de regressão obtidas entre a severidade real e a estimada (linha cheia) em doenças de final de ciclo (DFC) da soja (Glycine max). A linha pontilhada representa a situação ideal, com as estimativas idênticas à real.

a severidade estimada e a real, também chamada de erro absoluto ou resíduo (Figura 3).

Os maiores desvios ocorreram na faixa de $20 \%$ a $40 \%$ de severidade para a maioria dos avaliadores, sendo uma das possíveis razões para tal fato, a maior freqüência de halos amarelados nesses estádios de desenvolvimento da doença, o que pode confundir o avaliador menos experiente na definição da área doente. Entretanto, apenas algumas vezes os erros absolutos dos nove avaliadores ultrapassaram $15 \%$, concentrando-se na faixa inferior a 10\% (Figura 3). Esses valores dos resíduos são considerados bons quando se adota os critérios utilizados nos programas de computadores para treinamento de avaliadores de severidade de doenças, como Distrain (Tomerlin \& Howell, 1988)e Disease.Pro (Nutter Jr. \& Worawitlikit, 1989), os quais classificam como excelente um avaliador cujos erros não ultrapassam $5 \%$ e bom aquele cujos erros sejam menores que $10 \%$.

A maioria dos avaliadores superestimou a severidade das doenças avaliadas mas, segundo Godoy et al. (1997) e Nutter Jr. \& Schultz (1995), as soluções para corrigir a superestimativa da doença variam de acordo com a magnitude do erro, podendo ser corrigida com o treinamento dos avaliadores. Stonehouse
(1994) destacou que a presença de algum nível de erro absoluto nas mensurações pode ser compensada pela rapidez e padronização que resultam do uso de escalas diagramáticas.

Além da acurácia e da precisão, outro indicador da eficiência da escala diagramática desenvolvida é a reprodutibilidade das avaliações entre os avaliadores (Berger, 1980). Diferentes avaliadores, utilizando a mesma escala para a avaliação do mesmo material, devem estimar os mesmos valores de severidade (Nutter Jr. \& Schultz, 1995). Quando se compararam as estimativas feitas pelo avaliador $3 \mathrm{com}$ as dos demais, obteve-se altos coeficientes de determinação na maioria dos casos, variando de 0,77 a 0,91 . Neste caso, a severidade estimada pelo avaliador 3, explicou $84 \%$ da variação das avaliações do avaliador um, $88 \%$ do avaliador dois, $77 \%$ dos avaliadores quatro e cinco, $91 \%$ do avaliador seis, $87 \%$ do avaliador sete, $84 \%$ do avaliador oito e $87 \%$ do avaliador nove. Porém, nas estimativas dos avaliadores 5 e 8 observou-se maior discrepância entre suas estimativas quando comparadas com a do avaliador 3, pois nestes obteve-se coeficientes angulares baixos, 0,6 e 0,7, respectivamente. Essa situação pode ser resolvida com o treinamento dos avaliadores.

Os resultados obtidos pelos diversos avaliadores podem 
Escala diagramática para a quantificação do complexo de doenças...
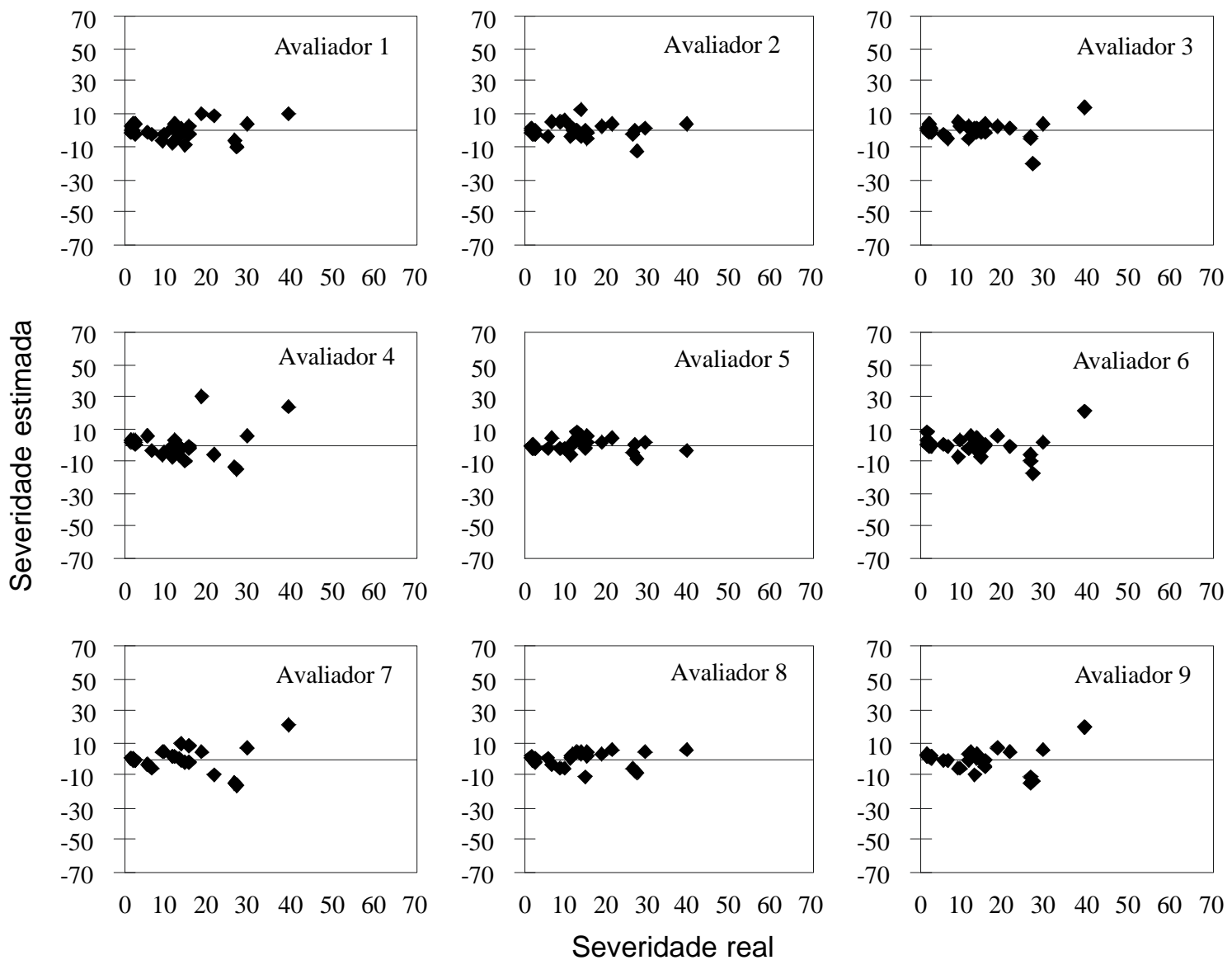

FIG. 3 - Erros absolutos (severidade estimada menos severidade real) para nove avaliadores de doenças de final de ciclo (DFC) da soja (Glycine max).

ser comparados entre si, fato que demonstra que a reprodutibilidade das avaliações foi garantida com o uso da escala diagramática desenvolvida.

O propósito de um sistema padrão para quantificação das DFC em soja é de grande responsabilidade, pois estimativas visuais imprecisas levam a erros que alteram conclusões de experimentos, conforme observado por Parker et al. (1995). A escala proposta neste trabalho apresenta-se como uma ferramenta bastante oportuna para quantificar a severidade dessas doenças, haja vista a falta de um sistema padrão de quantificação das mesmas.

\section{REFERÊNCIAS BIBLIOGRÁFICAS}

AMORIM, L. Avaliação de doenças. In: Bergamin Filho, A., Kimati, H., Amorim, L. (Eds.) Manual de fitopatologia, 3ed. São Paulo. Agronômica Ceres. 1995. pp.647-671.

AMORIM, L., BERGAMIN FILHO, A., PALAZZO, D., BASSANEZI, R.B., GODOY, C.V. \& TORRES, G.A.M. Clorose variegada dos citros: uma escala diagramática para avaliação da severidade da doença. Fitopatologia Brasileira 18:174-180. 1993.

BERGAMIN FILHO, A. \& AMORIM, L. Doenças de plantas tropicais: epidemiologia e controle econômico. Piracicaba. Agronômica Ceres. 1996.
BERGER, R.D. Measuring disease intensity. In: Teng, P.S. \& Krupa, S.V. (Eds.) Crop loss assessment. St. Paul. University of Minnesota. 1980. pp.28-31.

CAMPBELL, C.L. \& MADDEN, L.V. Introduction to plant disease epidemiology. New York. John Wiley. 1990.

EMBRAPA - EMPRESA BRASILEIRA DE PESQUISA AGROPECUÁRIA. Centro Nacional de Pesquisa de Soja. Recomendações técnicas para a cultura da soja na Região Central do Brasil 2000/01. Londrina. Embrapa-CNPSo. (Documentos, 146). 2000.

FAO - Organizacion de las Naciones Unidas para la agricultura y la alimentacion. El cultivo de la soja en los trópicos: mejoramiento y producion. Londrina. Embrapa-CNPSo. 1995.

FERREIRA, L.P., LEHMAN, P.S. \& ALMEIDA, A.M.R. Doenças da soja no Brasil. Londrina. . Embrapa-CNPSo. (Circular Técnica, 1). 1979 .

FORBES, G.A. \& KORVA, J.T. The effect of using Horsfall-Barratt scale on precision and accuracy of visual estimation of potato late blight severity in the field. Plant Pathology 43:675-682. 1994.

GODOY, C.V., CARNEIRO, S.M.T.P.G., IAMAUTI, M.T., DALLA PRIA, M., AMORIM, L., BERGER, R.D. \& BERGAMIN FILHO, A. Diagrammatic scales for bean diseases: development and validation. Zeitschrift für Pflanzenkrankheiten und Pflanzenschutz 104:336-345. 1997. 
HORSFALL, J.C. \& BARRAT, R.W. An improved grading system for measuring plant diseases. Phytopathology 35:665. 1945.

HORSFALL, J.G. \& COWLING, E.B. Pathometry: the measurement of plant disease. In: Horsfall, J.G. \& Cowling, E.B. (Eds.) Plant disease an advanced treatise. How disease develops in populations. New York. Academic Press. 1978. pp.119-136.

ITO, M.F. \& TANAKA, M.A.S. Soja: principais doenças causadas por fungos, bactérias e nematóides. Campinas. Fundação Cargill. (Série Técnica, 186). 1993.

LIM, S.M. Brown spot. In: Sinclair, J.B. \& Backman, P.A. (Eds.) Compendium of soybean diseases, 3.ed. Saint Paul. APS Press. 1989. pp.15-16.

NUTTER JR., F.W., GLEASON, M.L., JENCO, J.H. \& CHRISTIANS, N.C. Assessing the accuracy, intra-rater repeatability, and inter-rater reliability of disease assessment systems. Phytopathology 83:806-812. 1993.

NUTTER JR., F.W. \& SCHULTZ, P.M. Improving the accuracy and precision of disease assessments: selection of methods and use of computer-aided training programs. Canadian Journal of Plant Pathology 17:174-184. 1995.

NUTTER JR., F.W. \& WORAWITLIKIT, O. Disease.Pro: a computer program for evaluating and improving a person ability to assess disease proportion. Phytopathology 79:11135. 1989.

PARKER, S.R., SHAW, M.W. \& ROYLE, D.J. The reliability of visual estimates of disease severity on cereal leaves. Plant Pathology 44:856-864. 1995 .

STONEHOUSE, J. Assessment of Andean bean diseases using visual keys. Plant Pathology 43:519-527. 1994.

TOMERLIN, J.R. \& HOWELL, T.A. Distrain: a computer program for training people to estimate disease severity on cereal leaves. Plant Disease 72:455-459. 1988.

YORINORI, J.T. Controle integrado das principais doenças da soja. In: Câmara, G.M.S. (Ed.) Soja: tecnologia da produção. Piracicaba. Câmara, G.M.S. 1998. pp.139-192. 